\title{
Stability and bifurcation of Metzler equation
}

\section{Atena Ghasemabadi*}

\section{"Correspondence:}

ghasemabadi.math@gmail.com

Department of Mathematics,

University of Neyshabur, Neyshabur, Iran

\section{Introduction}

We consider the third order difference equation

$$
x_{n+1}=a x_{n}+b x_{n-1}+f\left(x_{n}-x_{n-1}\right)+g\left(x_{n-1}-x_{n-2}\right) \text {, }
$$

where $a, b \in(0,1), a+b<1$ and $f: \mathbb{R} \rightarrow \mathbb{R}, g: \mathbb{R} \rightarrow \mathbb{R}$ are the continuous real functions with $f(0)=0, g(0)=0, f(x) \neq 0, g(x) \neq 0$ for $x \neq 0$ and $x_{0}, x_{-1}, x_{-2}$ are given real numbers (initial conditions).

Metzler equation is a fundamental equation in economics. Particular cases of this equation have appeared in mathematical models of macroeconomics, see [1,2]. Equations of the form

$$
x_{n+1}=a x_{n}+f\left(x_{n}-x_{n-1}\right)
$$

are considered and studied extensively by [3-6].

In studying the behavior of the solutions of an equation, we often need to consider that its solutions are stable and attractive. Therefore, in Section 2, we study the relation of attractivity and stability of the equilibrium point of this equation and some related equations. In Section 3, we study the existence of Neimark-Sacker and period doubling (flip) bifurcation for this system by analyzing the characteristic equation. Furthermore, we investigate the direction of these bifurcations by using normal form theory.

(c) 2015 Ghasemabadi. This article is distributed under the terms of the Creative Commons Attribution 4.0 International License (http://creativecommons.org/licenses/by/4.0/), which permits unrestricted use, distribution, and reproduction in any medium, provided you give appropriate credit to the original author(s) and the source, provide a link to the Creative Commons license, and indicate if changes were made. 


\section{Attractivity}

In this section we study global attractivity and stability of the equilibrium point of (1.1). Equation (1.1) can be transformed to another form which has equivalent properties. Let

$$
u_{n}=x_{n}-x_{n-1}
$$

Then (1.1) is reduced to

$$
u_{n+1}=a u_{n}+b u_{n-1}+f\left(u_{n}\right)-f\left(u_{n-1}\right)+g\left(u_{n-1}\right)-g\left(u_{n-2}\right) \text {, }
$$

which has the unique equilibrium $\bar{u}=0$.

At first we show the following result.

Theorem 2.1 The equilibrium point $\bar{x}=0$ is globally attractive (respectively asymptotically stable) in (1.1) if and only if $\bar{u}=0$ is globally attractive (respectively asymptotically stable) in (2.2).

Proof Equation (1.1) can be written as

$$
x_{n+1}=(a+b) x_{n}-b u_{n}+f\left(u_{n}\right)+g\left(u_{n-1}\right) \quad \text { for } n=0,1,2, \ldots
$$

Hence we see that

$$
\begin{aligned}
x_{1}= & (a+b) x_{0}-b u_{0}+f\left(u_{0}\right)+g\left(u_{-1}\right), \\
x_{2}= & (a+b) x_{1}-b u_{1}+f\left(u_{1}\right)+g\left(u_{0}\right) \\
= & (a+b)^{2} x_{0}-b(a+b) u_{0}-b u_{1}+(a+b)\left(f\left(u_{0}\right)+g\left(u_{-1}\right)\right)+f\left(u_{1}\right)+g\left(u_{0}\right), \\
x_{3}= & (a+b) x_{2}-b u_{2}+f\left(u_{2}\right)+g\left(u_{1}\right) \\
= & (a+b)^{3} x_{0}-b(a+b)^{2} u_{0}-b(a+b) u_{1}-b u_{2}+(a+b)^{2}\left(f\left(u_{0}\right)+g\left(u_{-1}\right)\right) \\
& +(a+b)\left(f\left(u_{1}\right)+g\left(u_{0}\right)\right)+f\left(u_{2}\right)+g\left(u_{1}\right) .
\end{aligned}
$$

Using induction we have that

$$
x_{n}=(a+b)^{n} x_{0}-b \sum_{i=1}^{n}(a+b)^{n-i} u_{i-1}+\sum_{i=1}^{n}(a+b)^{n-i}\left(f\left(u_{i-1}\right)+g\left(u_{i-2}\right)\right) \text {, }
$$

let

$$
\bar{u}_{n}=\sum_{i=1}^{n}(a+b)^{n-i}\left|u_{i-1}\right|, \quad \bar{v}_{n}=\sum_{i=1}^{n}(a+b)^{n-i}\left|f\left(u_{i-1}\right)+g\left(u_{i-2}\right)\right| .
$$

We prove that

$$
\lim _{n \rightarrow \infty} \bar{u}_{n}=0, \quad \lim _{n \rightarrow \infty} \bar{v}_{n}=0 .
$$

We distinguish two cases for $\bar{u}_{n}$. 
Case 1. $\sum_{i=1}^{\infty}\left|u_{i-1}\right| /(a+b)^{i}<\infty$. In this case

$$
\lim _{n \rightarrow \infty} \bar{u}_{n}=\lim _{n \rightarrow \infty}(a+b)^{n} \sum_{i=1}^{n} \frac{\left|u_{i-1}\right|}{(a+b)^{i}}=0 .
$$

Case 2. $\sum_{i=1}^{\infty}\left|u_{i-1}\right| /(a+b)^{i}=\infty$. In this case, by using the Stolz theorem, we have that

$$
\begin{aligned}
\lim _{n \rightarrow \infty} \bar{u}_{n} & =\lim _{n \rightarrow \infty} \frac{\sum_{i=1}^{n} \frac{\left|u_{i-1}\right|}{(a+b)^{i}}}{\frac{1}{(a+b)^{n}}} \\
& =\lim _{n \rightarrow \infty} \frac{\sum_{i=1}^{n+1} \frac{\left|u_{i-1}\right|}{(a+b)^{i}}-\sum_{i=1}^{n} \frac{\left|u_{i-1}\right|}{(a+b)^{i}}}{\frac{1}{(a+b)^{n+1}}-\frac{1}{(a+b)^{n}}} \\
& =\lim _{n \rightarrow \infty} \frac{\left|u_{n}\right|}{1-(a+b)}=0 .
\end{aligned}
$$

Now we distinguish two cases for $\bar{v}_{n}$.

Case 1. $\sum_{i=1}^{\infty}\left|f\left(u_{i-1}\right)+g\left(u_{i-2}\right)\right| /(a+b)^{i}<\infty$. In this case

$$
\lim _{n \rightarrow \infty} \bar{v}_{n}=\lim _{n \rightarrow \infty}(a+b)^{n} \sum_{i=1}^{n} \frac{\left|f\left(u_{i-1}\right)+g\left(u_{i-2}\right)\right|}{(a+b)^{i}}=0 .
$$

Case 2. $\sum_{i=1}^{\infty}\left|f\left(u_{i-1}\right)+g\left(u_{i-2}\right)\right| /(a+b)^{i}=\infty$. In this case, by using the Stolz theorem, we have that

$$
\begin{aligned}
\lim _{n \rightarrow \infty} \bar{u}_{n} & =\lim _{n \rightarrow \infty} \frac{\sum_{i=1}^{n} \frac{\left|f\left(u_{i-1}\right)+g\left(u_{i-2}\right)\right|}{(a+b)^{i}}}{\frac{1}{(a+b)^{n}}} \\
& =\lim _{n \rightarrow \infty} \frac{\sum_{i=1}^{n+1} \frac{\left|f\left(u_{i-1}\right)+g\left(u_{i-2}\right)\right|}{(a+b)^{i}}-\sum_{i=1}^{n} \frac{\left|f\left(u_{i-1}\right)+g\left(u_{i-2}\right)\right|}{(a+b)^{i}}}{\frac{1}{(a+b)^{n+1}}-\frac{1}{(a+b)^{n}}} \\
& =\lim _{n \rightarrow \infty} \frac{\left|f\left(u_{n}\right)+g\left(u_{n-1}\right)\right|}{1-(a+b)} .
\end{aligned}
$$

Using the continuity of $f, g$ we see that if $\lim _{n \rightarrow \infty} u_{n}=0$ then $\lim _{n \rightarrow \infty} \bar{u}_{n}=0, \lim _{n \rightarrow \infty} \bar{v}_{n}=$ 0 , which implies that $\lim _{n \rightarrow \infty} x_{n}=0$.

By using weak contractions introduced in [6], we obtain the following sufficient conditions for attractivity of solutions of (1.1).

\section{Proposition 2.2}

(1) If $|f(t)+g(s)| \leq c|t+s|$ for all $t$, $s$ and $0<c<\frac{1-(a+b)}{2}$, then the origin is globally attractive in (1.1).

(2) If $0 \leq f(t)+g(s) \leq c|t+s|$ for all $t$, $s$ and $0<c<1-(a+b)$, then every positive solution of (1.1) converges to zero.

Proof For the proof of (1), define $F\left(y_{1}, y_{2}, y_{3}\right)=a y_{3}+b y_{2}+f\left(y_{3}-y_{2}\right)+g\left(y_{2}-y_{1}\right)$ and notice that

$$
\begin{aligned}
\left|F\left(y_{1}, y_{2}, y_{3}\right)\right| & \leq a\left|y_{3}\right|+b\left|y_{2}\right|+\left|f\left(y_{3}-y_{2}\right)+g\left(y_{2}-y_{1}\right)\right| \\
& \leq a\left|y_{3}\right|+b\left|y_{2}\right|+c\left|y_{3}-y_{1}\right| \\
& \leq(a+b+2 c) \max \left\{\left|y_{1}\right|,\left|y_{2}\right|,\left|y_{3}\right|\right\} .
\end{aligned}
$$


Since $a+b+2 c<1$, it follows that $F$ is a weak contraction on the entire space, and therefore by [6], the origin is globally attractive.

(2) For $y_{1}, y_{2}, y_{3} \geq 0$, notice that

$$
\begin{aligned}
F\left(y_{1}, y_{2}, y_{3}\right) & \leq a y_{3}+b y_{2}+f\left(y_{3}-y_{2}\right)+g\left(y_{2}-y_{1}\right) \\
& \leq a y_{3}+b y_{2}+c\left|y_{3}-y_{1}\right| \\
& \leq(a+b+c) \max \left\{y_{1}, y_{2}, y_{3}\right\} .
\end{aligned}
$$

Now, since $a+b+c<1$, it follows that $F$ is a weak contraction on $[0, \infty)^{3}$, and since $[0, \infty)^{3}$ is invariant under $V_{F}\left(y_{1}, y_{2}, y_{3}\right)=\left(F\left(y_{1}, y_{2}, y_{3}\right), y_{1}, y_{2}\right)$, [6] implies that the origin is exponentially stable relative to $[0, \infty)^{3}$, hence every positive solution of $(1.1)$ converges to zero.

Now we study stability properties. Let $x_{n}$ be a solution of (1.1). We define the vector $y(n)=\left(y_{1}(n), y_{2}(n), y_{3}(n)\right) \in \mathbb{R}^{3}$, where

$$
y_{j}(n)=x_{n+j-3}, \quad j=1,2,3 .
$$

Using this notation the delay equation (1.1) is transformed to the following 3D system:

$$
y(n+1)=h(y(n))
$$

where $h(y)=\left(y_{2}, y_{3}, a y_{3}+b y_{2}+f\left(y_{3}-y_{2}\right)+g\left(y_{2}-y_{1}\right)\right)$, which has the unique equilibrium point $(0,0,0)$.

Now we study the relation of the stability properties of the delay equation (1.1) to those of the associated nondelay equation

$$
x_{n+1}=f\left(x_{n}\right), \quad n \geq-1 .
$$

First we prove the following lemma which will be used in the next results.

Lemma 2.3 Let $y(n)$ be a solution of system (2.7). Then, for $n \geq 4-j$, the following statements are true:

$$
\begin{aligned}
\left|y_{j}(n)\right| \leq & a^{n+j-3}\left|y_{3}(0)\right|+b \sum_{i=1}^{n+j-3} a^{n+j-i-3}\left|y_{2}(i-1)\right| \\
& +\sum_{i=1}^{n+j-3} a^{n+j-i-3}\left|f\left(y_{3}(i-1)-y_{2}(i-1)\right)\right| \\
& +\sum_{i=1}^{n+j-3} a^{n+j-i-3}\left|g\left(y_{2}(i-1)-y_{1}(i-1)\right)\right| .
\end{aligned}
$$

Furthermore, for $0 \leq n \leq 3-j$,

$$
y_{j}(n)=y_{j+n}(0) .
$$


Proof From (2.5) we have that for $j=1,2,3$,

$$
\begin{aligned}
& y_{j}(n)=x_{n+j-3}=x_{(n-1)+(j+1)-3}=y_{j+1}(n-1), \\
& y_{j}(n)=x_{n+j-3}=x_{0+(n+j)-3}=y_{n+j}(0) .
\end{aligned}
$$

Now, by using these relations and induction, we see that

$$
\begin{aligned}
y_{3}(n)= & a^{n} y_{3}(0)+b \sum_{i=1}^{n} a^{n-i} y_{2}(i-1) \\
& +\sum_{i=1}^{n} a^{n-i} f\left(y_{3}(i-1)-y_{2}(i-1)\right)+\sum_{i=1}^{n} a^{n-i} g\left(y_{2}(i-1)-y_{1}(i-1)\right) .
\end{aligned}
$$

Furthermore,

$$
y_{j}(n)=x_{n+j-3}=x_{(n+j-3)+3-3}=y_{3}(n+j-3) .
$$

By using this relation, we have that

$$
\begin{aligned}
y_{j}(n)= & y_{3}(n+j-3) \\
= & a^{n+j-3} y_{3}(0)+b \sum_{i=1}^{n+j-3} a^{n+j-i-3} y_{2}(i-1) \\
& +\sum_{i=1}^{n+j-3} a^{n+j-i-3} f\left(y_{3}(i-1)-y_{2}(i-1)\right) \\
& +\sum_{i=1}^{n+j-3} a^{n+j-i-3} g\left(y_{2}(i-1)-y_{1}(i-1)\right) .
\end{aligned}
$$

Theorem 2.4 Assume that $f$, gatisfy

$$
|f(x+y)| \leq|f(x)|+|f(y)|, \quad|g(x)| \leq c|x|
$$

for all $x, y \in \mathbb{R}, c<1$. If the equilibrium point of (2.7) is stable, then the equilibrium point of (1.1) is also stable.

Proof It is sufficient to prove the stability of the equilibrium of (2.6) because of the equivalence of (1.1) and (2.6). Let $\varepsilon>0$ be arbitrary. Since the equilibrium point of (2.7) is stable, there exists $\delta_{1}>0$ such that $\left|x_{-2}\right|<\delta_{1}$ implies $\left|x_{n}\right|<\frac{(1-a) \varepsilon}{8}$ for all $n \geq-2$. Choose $\delta=\min \left(\delta_{1}, \frac{(1-a) \varepsilon}{8}\right)$, since $y(0)=\left(y_{1}(0), y_{2}(0), y_{3}(0)\right)=\left(x_{-2}, x_{-1}, x_{0}\right)$, we have that

$$
\|y(0)\|=\max \left(\left|y_{1}(0)\right|,\left|y_{2}(0)\right|,\left|y_{3}(0)\right|\right)=\max \left(\left|x_{-2}\right|,\left|x_{-1}\right|,\left|x_{0}\right|\right) \leq \delta \leq \delta_{1} .
$$

Now, for $n \geq-2$,

$$
\left|x_{n}\right| \leq \frac{(1-a) \varepsilon}{8}
$$


which implies that

$$
\left|f\left(x_{n}\right)\right| \leq \frac{(1-a) \varepsilon}{8}
$$

for all $n \geq-1$. Therefore, for $n \geq 0$,

$$
\left|f\left(y_{3}(n-1)\right)\right| \leq \frac{(1-a) \varepsilon}{8}, \quad\left|f\left(y_{2}(n-1)\right)\right| \leq \frac{(1-a) \varepsilon}{8} .
$$

And hence

$$
\begin{aligned}
\left|f\left(y_{3}(n-1)-y_{2}(n-1)\right)\right| & <\left|f\left(y_{3}(n-1)\right)\right|+\left|f\left(y_{2}(n-1)\right)\right| \\
& <\frac{(1-a) \varepsilon}{8}+\frac{(1-a) \varepsilon}{8}=\frac{(1-a) \varepsilon}{4} .
\end{aligned}
$$

Now $\|y(0)\| \leq \delta$ implies that $\left|y_{j}(0)\right|<\delta<\frac{(1-a) \varepsilon}{8}<\varepsilon$ for $j=1,2,3$. Hence

$$
\left|y_{j}(n)\right|=\left|y_{j+n}(0)\right|<\varepsilon \quad \text { for } 0 \leq n \leq 3-j,
$$

and from the previous lemma we have

$$
\begin{aligned}
\left|y_{j}(n)\right| \leq & a^{n+j-3}\left|y_{3}(0)\right|+b \sum_{i=1}^{n+j-3} a^{n+j-i-3}\left|y_{2}(i-1)\right| \\
& +\sum_{i=1}^{n+j-3} a^{n+j-i-3}\left|f\left(y_{3}(i-1)-y_{2}(i-1)\right)\right| \\
& +\sum_{i=1}^{n+j-3} a^{n+j-i-3}\left|g\left(y_{2}(i-1)-y_{1}(i-1)\right)\right| \\
< & a^{n+j-3} \varepsilon+\frac{b(1-a) \varepsilon}{8} a^{n+j-3} \sum_{i=1}^{n+j-3} a^{-i} \\
& +\frac{(1-a) \varepsilon}{4} a^{n+j-3} \sum_{i=1}^{n+j-3} a^{-i}+\frac{c(1-a) \varepsilon}{4} a^{n+j-3} \sum_{i=1}^{n+j-3} a^{-i} \\
< & a^{n+j-3} \varepsilon+\frac{b \varepsilon}{8}\left(1-a^{n+j-3}\right) \\
& +\frac{\varepsilon}{4}\left(1-a^{n+j-3}\right)+\frac{c \varepsilon}{4}\left(1-a^{n+j-3}\right) \\
< & a^{n+j-3} \varepsilon+\varepsilon\left(1-a^{n+j-3}\right)=\varepsilon .
\end{aligned}
$$

Therefore, for arbitrary $\varepsilon>0$, there exists $\delta>0$ such that $\|y(0)\|<\delta$ implies that $\|y(n)\|<\varepsilon$ for $n \geq 0$, and hence the equilibrium point of (2.6) is stable.

\section{Existence of bifurcations}

Now we study bifurcations of (1.1), for this aim we suppose that $f, g \in C^{3}$. First we prove the existence of bifurcations. Dynamics of system (1.1) is described by the mapping

$$
h\left(y_{1}, y_{2}, y_{3}\right)=\left(y_{2}, y_{3}, a y_{3}+b y_{2}+f\left(y_{3}-y_{2}\right)+g\left(y_{2}-y_{1}\right)\right) .
$$


The Jacobian matrix of $h$ at $O$ is

$$
H=D h_{\left.\right|_{O}}=\left[\begin{array}{ccc}
0 & 1 & 0 \\
0 & 0 & 1 \\
-q & n & m
\end{array}\right] \text {, }
$$

in which $n=b-p+q, m=a+p$ that $p=f^{\prime}(0), q=g^{\prime}(0)$. The characteristic equation of $H$ is

$$
\lambda^{3}-m \lambda^{2}-n \lambda+q=0
$$

By Jury's conditions, the necessary and sufficient conditions for all eigenvalues of the characteristic equation (3.2) lying inside the unit circle are as follows:

$$
\left\{\begin{array}{l}
m-n+1-q>0 \\
q-m-n+1>0 \\
|q|<1 \\
n-m q-q^{2}+1>0 \\
m q-n-q^{2}+1>0
\end{array}\right.
$$

We consider the following curves:

$$
\left\{\begin{array}{l}
L_{1}: m-n+1-q=0, \\
L_{2}: n-m q-q^{2}+1=0, \quad q>0 \\
L_{3}: q-m-n+1=0 \\
L_{4}:|q|=1
\end{array}\right.
$$

On $L_{3}, a+b=1$, which is impossible.

Wen et al. in [7, 8] proved the following two lemmas which will be used in the next theorem.

Lemma 3.1 In the characteristic polynomial equation $\lambda^{3}+a_{2} \lambda^{2}+a_{1} \lambda+a_{0}=0$, one of the eigenvalues will be -1 and other eigenvalues will be inside the unit circle if and only if the following relations hold:

(b1) $\left|a_{0}\right|<1$,

(b2) $a_{0}+a_{2}=1+a_{1}$,

(b3) $\left|a_{1}-a_{0} a_{2}\right|<1-a_{0}^{2}$.

Lemma 3.2 The characteristic polynomial equation $\lambda^{3}+a_{2} \lambda^{2}+a_{1} \lambda+a_{0}=0$ has a pair of complex conjugate lying on the unit circle, and the third eigenvalue lies inside the unit circle if and only if the following relations hold:

(c1) $\left|a_{0}\right|<1$,

(c2) $\left|a_{0}+a_{2}\right|<1+a_{1}$,

(c3) $a_{1}-a_{0} a_{2}=1-a_{0}^{2}$.

We show in the following theorem the occurrence of bifurcations on the curves $L_{1}, L_{2}$. 
Theorem 3.3 For system (3.1), the following conditions hold:

(1) Flip bifurcation occurs when $(m, n, q) \in L_{1}$ and $|q|<1$ and $|m q-n|<1-q^{2}$.

(2) Neimark-Sacker bifurcation occurs when $(m, n, q) \in L_{2}$ and $p, q>0$ and $|q|<1$ and $|q-m|<1-n$.

Proof First, we show the existence of flip bifurcation. Because $(m, n, q) \in L_{1}$, we have the characteristic equation

$$
(\lambda+1)\left(\lambda^{2}-(1+m) \lambda+q\right)=0,
$$

which has eigenvalues $\lambda_{1}=-1, \lambda_{2,3}=\left(\frac{1+m \pm \sqrt{(1+m)^{2}-4 q}}{2}\right)$. So $Y=(1,-1,1)$ is an eigenvector of $H$ with corresponding eigenvalue $\lambda_{1}=-1$, and 1 is not the eigenvalue. A straightforward calculation shows that

$$
\operatorname{Range}\left(I+D h\left(X^{*}\right)\right)_{\mid m-n+1-q=0}=\operatorname{Span}(x+y, y+z,-q x+n y+(1+m) z)^{T},
$$

in which $(x, y, z) \in \mathbb{R}^{3}$. Now

$$
\frac{d}{d q} D h\left(X^{*}\right)_{\mid m-n+1-q=0}=\left[\begin{array}{ccc}
0 & 0 & 0 \\
0 & 0 & 0 \\
-1 & 1 & 0
\end{array}\right]
$$

and

$$
\frac{d}{d q} \operatorname{Dh}\left(X^{*}\right)_{\left.\right|_{m-n+1-q=0}} Y=(0,0,-2) \notin \operatorname{Range}\left(I+D g\left(X^{*}\right)\right)_{\left.\right|_{m-n+1-q=0}} .
$$

Therefore by Theorem 1.4.5 of [9], the flip bifurcation occurs.

Now we show the existence of Neimark-Sacker bifurcation. Because $(m, n, q) \in L_{2}$, we have

$$
(\lambda+q)\left(\lambda^{2}-(m+q) \lambda+1\right)=0 .
$$

By using Lemma 3.2, the characteristic equation (3.5) has complex conjugate roots $\lambda_{2,3}=$ $e^{ \pm i \theta}$, therefore $\Delta=(m+q)^{2}-4<0$ or $|m+q|<2$.

By separating the real and imaginary parts, we have

$$
\left\{\begin{array}{l}
\cos 2 \theta-(m+q) \cos \theta=-1, \\
\sin 2 \theta-(m+q) \sin \theta=0 .
\end{array}\right.
$$

Squaring and adding both equations, we have

$$
\cos \theta=\frac{m+q}{2} .
$$

Since $f, g$ are investment functions, we can assume that $p=f^{\prime}(0)>0, q=g^{\prime}(0)>0$; therefore, $a+p+q=m+q>0$ or $0<m+q<2$ and we obtain

$$
0<\cos \theta=\frac{m+q}{2}<1 \text {, }
$$

which refers to $\arg \lambda \neq 0, \pm \frac{\pi}{2}, \pm \frac{2 \pi}{3}, \pm \pi$. Thus $\lambda^{k} \neq 1$ for $k=1,2,3$ and 4 . 
On the other hand, we have

$$
\begin{aligned}
\left(\frac{d|\lambda|^{2}}{d q}\right)_{\left.\right|_{n-m q-q^{2}+1=0}} & =\left(\bar{\lambda} \frac{d \lambda}{d q}+\lambda \frac{d \bar{\lambda}}{d q}\right)_{\left.\right|_{n-m q-q^{2}+1=0}} \\
& =\frac{2(-3 \cos 3 \theta+(2 m+3) \cos 2 \theta+(n-2 m) \cos \theta-n)}{\left|3 e^{2 i \theta}-2 m e^{i \theta}-n\right|^{2}} .
\end{aligned}
$$

Also, $\cos 3 \theta=4 \cos ^{3} \theta-3 \cos \theta$ and $\cos 2 \theta=2 \cos ^{2} \theta-1$, hence we have

$$
\begin{aligned}
& -3 \cos 3 \theta+(2 m+3) \cos 2 \theta+(n-2 m) \cos \theta-n \\
& \quad=-12 \cos ^{3} \theta+2(2 m+3) \cos ^{2} \theta+(n-2 m+9) \cos \theta-2 m-n-3 .
\end{aligned}
$$

Hence

$$
\left(\frac{d|\lambda|^{2}}{d q}\right)_{\left.\right|_{n-m q-q^{2}+1=0}}=\frac{2(1-\cos \theta)\left(12 \cos ^{2} \theta-(4 m-6) \cos \theta-(n+2 m+3)\right)}{\left|3 e^{2 i \theta}-2 m e^{i \theta}-n\right|^{2}} .
$$

Assume that $\left(\frac{d|\lambda|^{2}}{d q}\right)_{\left.\right|_{n-m q-q^{2}+1=0}}=0$, that is, $\cos \theta=1$ or $12 \cos ^{2} \theta-(4 m-6) \cos \theta-(n+$ $2 m+3)=0$. In the previous discussion, we have obtained $0<\cos \theta<1$, hence $\cos \theta \neq 1$. In $12 \cos ^{2} \theta-(4 m-6) \cos \theta-(n+2 m+3)$, we substitute $\frac{m+q}{2}$ instead of $\cos \theta$, and we obtain

$$
12 \cos ^{2} \theta-(4 m-6) \cos \theta-(n+2 m+3)=m^{2}+3 q^{2}+4 m q+m+3 q-n-3 .
$$

Case $1.1<m+q<2$. In equation (3.7), we replace $-n-1$ with $-q^{2}-m q$ and $q^{2}+m q-1$ with $n$, then we obtain

$$
m^{2}+3 q^{2}+4 m q+m+3 q-n-3=(m+q)^{2}-1+m+n+3 q .
$$

Since $m+n+3 q=a+b+4 q>0$, then we obtain

$$
0<(m+q)^{2}-1+m+n+3 q .
$$

Thus, in the case $1<m+q<2$, we get that $\left(\frac{d|\lambda|^{2}}{d q}\right)_{\left.\right|_{n-m q-q^{2}+1=0}} \neq 0$.

Case 2. $0<m+q \leq 1$. We claim that $m^{2}+3 q^{2}+4 m q+m+3 q-n-3 \neq 0$.

We consider $m^{2}+3 q^{2}+4 m q+m+3 q-n-3=0$ and define $h=b-p+1$,

$$
0=m^{2}+3 q^{2}+4 m q+m+3 q-n-3=(m+q)^{2}+2 q(m+q)+(m+q)+q-h-2 .
$$

Since $q>0$ and $(m, n, q) \in L_{2}$, i.e., $q(m+q)=n+1=h+q$, we have

$$
m+q=\frac{h+q}{q}
$$

We substitute $\frac{h+q}{q}$ instead of $m+q$ and obtain

$$
\begin{aligned}
0 & =m^{2}+3 q^{2}+4 m q+m+3 q-n-3=(m+q)^{2}+2 q(m+q)+(m+q)+q-h-2 \\
& =\left(\frac{h+q}{q}\right)^{2}+2 q\left(\frac{h+q}{q}\right)+\left(\frac{h+q}{q}\right)+q-h-2,
\end{aligned}
$$


thus

$$
0=(h+q)^{2}+2 q^{2}(h+q)+q(h+q)+q^{3}-q^{2} h-2 q^{2}=h^{2}+q^{2} h+3 q h+3 q^{3},
$$

hence

$$
h^{2}+3 q^{3}+h\left(3 q+q^{2}\right)=0
$$

Since $q>0$, we have

$$
h^{2}+3 q^{3}>0, \quad 3 q+q^{2}>0
$$

therefore $h<0$ or $b-p+1<0$,

$$
\begin{aligned}
& b-p+1<0 \quad \Longrightarrow \quad p>b+1>1 \\
& \Longrightarrow \quad a+p+q>1 \\
& \Longrightarrow \quad m+q>1 \text {. }
\end{aligned}
$$

Thus if $m^{2}+3 q^{2}+4 m q+m+3 q-n-3=0$, then $m+q>1$.

Using contrapositive law, we get if $m+q \leq 1$, then $m^{2}+3 q^{2}+4 m q+m+3 q-n-3 \neq 0$.

From the above discussion it can be inferred that if $1<m+q<2$, then $m^{2}+3 q^{2}+4 m q+$ $m+3 q-n-3 \neq 0$ and if $m+q \leq 1$, then $m^{2}+3 q^{2}+4 m q+m+3 q-n-3 \neq 0$. Thus

$$
\left(\frac{d|\lambda|^{2}}{d q}\right)_{\left.\right|_{n-m q-q^{2}+1=0}} \neq 0
$$

Therefore, by the generic Neimark-Sacker bifurcation theorem [10, 11], the NeimarkSacker bifurcation occurs, that is, system (3.1) has a unique closed invariant curve bifurcating from the equilibrium $X^{*}$.

\section{Direction of the bifurcations}

In the previous section, we have shown that system (3.1) undergoes a flip (period-doubling) bifurcation when $(p, q) \in L_{1}$ and a Neimark-Sacker bifurcation when $(p, q) \in L_{3}$ at the equilibrium point $X^{*}$. In this section, by using the normal form method for discrete systems, as studied by Sacker, Kuznetsov and Wiggins, we shall study the direction of the two bifurcations and stability of the bifurcating invariant curves. We can write the system as

$$
U_{n+1}=D U_{n}+G\left(U_{n}\right), \quad U_{n} \in \mathbb{R}^{3},
$$

where $G(U)=O\left(\|U\|^{3}\right)$ is a smooth function and its Taylor expansion is

$$
G(U)=\frac{1}{2} B(U, U)+\frac{1}{6} C(U, U, U)+O\left(\|U\|^{3}\right),
$$

where

$$
B(U, U)=\left(0,0, b_{3}(U, U)\right), \quad C(U, U, U)=\left(0,0, c_{3}(U, U, U)\right),
$$


in which

$$
\left\{\begin{aligned}
& b_{3}(\phi, \psi)=f^{\prime \prime}(0)\left(\phi_{2} \psi_{2}-\phi_{2} \psi_{3}+\phi_{3} \psi_{3}-\phi_{3} \psi_{2}\right) \\
&+ g^{\prime \prime}(0)\left(\phi_{1} \psi_{1}-\phi_{2} \psi_{1}-\phi_{1} \psi_{2}+\phi_{2} \psi_{2}\right), \\
& c_{3}(\phi, \psi, \eta)= g^{\prime \prime \prime}(0)\left(\phi_{1} \psi_{2} \eta_{1}-\phi_{1} \psi_{2} \eta_{2}-\phi_{1} \psi_{1} \eta_{1}+\phi_{1} \psi_{1} \eta_{2}\right. \\
&\left.+\phi_{2} \psi_{2} \eta_{2}-\phi_{2} \psi_{2} \eta_{1}+\phi_{2} \psi_{1} \eta_{1}-\phi_{2} \psi_{1} \eta_{2}\right) \\
&+f^{\prime \prime \prime}(0)\left(\phi_{2} \psi_{2} \eta_{3}-\phi_{2} \psi_{2} \eta_{2}+\phi_{3} \psi_{3} \eta_{3}-\phi_{3} \psi_{3} \eta_{2}\right. \\
&\left.-\phi_{3} \psi_{2} \eta_{3}+\phi_{3} \psi_{2} \eta_{2}-\phi_{2} \psi_{3} \eta_{3}+\phi_{2} \psi_{3} \eta_{2}\right) .
\end{aligned}\right.
$$

For $\phi=\left(\phi_{1}, \phi_{2}, \phi_{3}\right) \in \mathbb{R}^{3}, \psi=\left(\psi_{1}, \psi_{2}, \psi_{3}\right) \in \mathbb{R}^{3}$ and $\eta=\left(\eta_{1}, \eta_{2}, \eta_{3}\right) \in \mathbb{R}^{3}$. At the beginning, we study the direction of period-doubling bifurcation and the stability of period-doubling cycle. Let $w \in \mathbb{R}^{3}$ be the eigenvector of $H$ with respect to eigenvalue -1 , that is, $H w=-w$; let $v \in \mathbb{R}^{3}$ be the adjoint eigenvector of $H^{T}$, that is, $H^{T} v=-v$, where $H^{T}$ is the transposed matrix, and $\langle v, w\rangle=1$, where $\langle\cdot, \cdot\rangle$ is the standard scalar product in $\mathbb{R}^{3}$. So we have

$$
\left\{\begin{array}{l}
w=(-1,1,-1), \\
v=\frac{-1}{2+m+q}(q,-(1+m), 1) .
\end{array}\right.
$$

Following the algorithms given in Kuznetsov [10], the critical normal form coefficient $c(0)$, that determines the nondegeneracy of period-doubling bifurcation and the stability of period-doubling cycle, is given by the following formula:

$$
c(0)=\frac{1}{6}\langle v, C(w, w, w)\rangle-\frac{1}{2}\left\langle v, B\left(w,(H-I)^{-1} B(w, w)\right)\right\rangle .
$$

From the above relations we have

$$
\left\{\begin{array}{l}
\left\langle v, B\left(w,(H-I)^{-1} B(w, w)\right)\right\rangle=0, \\
\langle v, C(w, w, w)\rangle=\frac{-8 f^{\prime \prime \prime}(0)+8 g^{\prime \prime \prime}(0)}{2+m+q},
\end{array}\right.
$$

and therefore

$$
c(0)=\frac{1}{3} \frac{-4 f^{\prime \prime \prime}(0)+4 g^{\prime \prime \prime}(0)}{2+m+q} .
$$

Applying the general theory for the direction of flip bifurcation and the stability of period doubling cycle (see Wiggins [11] or Kuznetsov [10]), we derive the following result.

Theorem 4.1 For system (3.1), a flip bifurcation occurs in $X^{*}$ when $m-n+1-q=0$, and if $f^{\prime \prime \prime}(0)<g^{\prime \prime \prime}(0)$, the flip bifurcation is supercritical and if $f^{\prime \prime \prime}(0)>g^{\prime \prime \prime}(0)$, the flip bifurcation is subcritical.

Now, we are going to study the direction of the Neimark-Sacker bifurcation and the stability of the bifurcating invariant curve in $X^{*}$. In the above section, we see that $H$ has simple eigenvalues on the unit circle

$$
\lambda_{1,2}=e^{ \pm i \theta_{0}}, \quad \theta_{0}=\arccos \frac{m+q}{2} .
$$


Let $\alpha$ be a complex eigenvector corresponding to $e^{i \theta}$ and $\beta$ be a complex eigenvector of the transposed matrix $H^{T}$ corresponding to $e^{-i \theta}$, i.e., $H \alpha=e^{i \theta_{0}} \alpha, H^{T} \beta=e^{-i \theta_{0}} \beta$. By computation we obtain the following eigenvectors:

$$
\alpha=\left(1, e^{i \theta_{0}}, e^{2 i \theta_{0}}\right)^{T}, \quad \beta=\frac{1}{q}\left(q, m e^{-i \theta_{0}}-e^{-2 i \theta_{0}},-e^{-i \theta_{0}}\right)^{T} .
$$

Normalize $\alpha$ with respect to $\beta$ such that

$$
\langle\beta, \alpha\rangle=1,
$$

where $\langle\cdot, \cdot \cdot\rangle$ means the standard scalar product in $\mathbb{C}^{3}$ defined by $\langle\beta, \alpha\rangle=\overline{\beta_{1}} \alpha_{1}+\overline{\beta_{2}} \alpha_{2}+\overline{\beta_{3}} \alpha_{3}$, we have

$$
\alpha=\left(1, e^{i \theta_{0}}, e^{2 i \theta_{0}}\right)^{T}, \quad \beta=\frac{1}{q+m e^{2 i \theta_{0}}-2 e^{3 i \theta_{0}}}\left(q, m e^{-i \theta_{0}}-e^{-2 i \theta_{0}},-e^{-i \theta_{0}}\right)^{T} .
$$

Following the algorithms given in Kuznetsov [10], the critical normal form coefficient $a(0)$, that determines the nondegeneracy of Neimark-Sacker bifurcation and allows us to predict the stability of bifurcating invariant curve, is given by the following formula:

$$
\begin{aligned}
a(0)= & \frac{1}{2} \operatorname{Re}\left(e ^ { - i \theta _ { 0 } } \left[\langle\beta, C(\alpha, \alpha, \bar{\alpha})\rangle+2\left\langle\beta, B\left(\alpha,(I-H)^{-1} B(\alpha, \bar{\alpha})\right)\right\rangle\right.\right. \\
& \left.\left.+\left\langle\beta, B\left(\bar{\alpha},\left(e^{2 i \theta_{0}} I-H\right)^{-1}\right) B(\alpha, \alpha)\right\rangle\right]\right) .
\end{aligned}
$$

Furthermore, in this case we have

$$
\begin{aligned}
& \langle\beta, C(\alpha, \alpha, \bar{\alpha})\rangle=\frac{f^{\prime \prime \prime}(0)\left(-e^{i \theta_{0}}+3 e^{2 i \theta_{0}}-3 e^{3 i \theta_{0}}+e^{4 i \theta_{0}}\right)+g^{\prime \prime \prime}(0)\left(3 e^{i \theta_{0}}-3 e^{2 i \theta_{0}}-1+e^{3 i \theta_{0}}\right)}{q+m e^{-2 i \theta_{0}}-2 e^{-3 i \theta_{0}}}, \\
& \left\langle\beta, B\left(\alpha,(I-H)^{-1} B(\alpha, \bar{\alpha})\right)\right\rangle=0, \\
& \left\langle\beta, B\left(\bar{\alpha},\left(e^{2 i \theta_{0}} I-H\right)^{-1}\right) B(\alpha, \alpha)\right\rangle \\
& \quad=\frac{\left(g^{\prime \prime}(0)\right)^{2}\left(-3 e^{i \theta_{0}}+1+2 e^{2 i \theta_{0}}+2 e^{3 i \theta_{0}}-3 e^{4 i \theta_{0}}+e^{5 i \theta_{0}}\right)}{\left(e^{6 i \theta_{0}}-m e^{4 i \theta_{0}}-n e^{2 i \theta_{0}}+q\right)\left(q+m e^{-2 i \theta_{0}}-2 e^{-3 i \theta_{0}}\right)} \\
& \quad+\frac{\left(f^{\prime \prime}(0)\right)^{2}\left(-3 e^{4 i \theta_{0}}+2 e^{5 i \theta_{0}}+2 e^{6 i \theta_{0}}+e^{3 i \theta_{0}}-3 e^{7 i \theta_{0}}+e^{8 i \theta_{0}}\right)}{\left(e^{6 i \theta_{0}}-m e^{4 i \theta_{0}}-n e^{2 i \theta_{0}}+q\right)\left(q+m e^{-2 i \theta_{0}}-2 e^{-3 i \theta_{0}}\right)} \\
& \quad+\frac{\left(f^{\prime \prime}(0) g^{\prime \prime}(0)\right)\left(e^{i \theta_{0}}-2 e^{2 i \theta_{0}}-e^{3 i \theta_{0}}+4 e^{4 i \theta_{0}}-e^{5 i \theta_{0}}-2 e^{6 i \theta_{0}}+e^{7 i \theta_{0}}\right)}{\left(e^{6 i \theta_{0}}-m e^{4 i \theta_{0}}-n e^{2 i \theta_{0}}+q\right)\left(q+m e^{-2 i \theta_{0}}-2 e^{-3 i \theta_{0}}\right)}
\end{aligned}
$$

which yields the following formula for $a(0)$ :

$$
a(0)=\frac{f^{\prime \prime \prime}(0) A_{1}+g^{\prime \prime \prime}(0) A_{2}+\left(g^{\prime \prime}(0)\right)^{2} A_{3}+\left(f^{\prime \prime}(0)\right)^{2} A_{4}+f^{\prime \prime}(0) g^{\prime \prime}(0) A_{5}}{A_{0}^{2}+B_{0}^{2}},
$$

in which

$$
\begin{aligned}
A_{0}= & q \cos 6 \theta_{0}+(m-m q) \cos 4 \theta_{0}-2 \cos 3 \theta_{0} \\
& -\left(m^{2}+n q-m q\right) \cos 2 \theta_{0}+(2 m+n-2 q) \cos \theta_{0},
\end{aligned}
$$




$$
\begin{aligned}
& B_{0}=q \sin 6 \theta_{0}+(m-m q) \sin 4 \theta_{0}-2 \sin 3 \theta_{0} \\
& -\left(m^{2}+n q-m q\right) \sin 2 \theta_{0}+(2 m+n-2 q) \sin \theta_{0}, \\
& A_{1}=(6 q+m q+3) \cos 5 \theta_{0}-3 q \cos 6 \theta_{0}+(2 n-2 m q-9 q-m) \cos 4 \theta_{0} \\
& +(3+3 m+2 q-6 n) \cos 3 \theta_{0}+\left(m^{2}+n q-6-m+2 m q+6 n\right) \cos 2 \theta_{0} \\
& +\left(6-4 m^{2}-4 n q-7 m-m q\right) \cos \theta_{0}+\left(6 m+3 m^{2}+3 n q-2\right), \\
& A_{2}=3 q \cos 6 \theta_{0}-q \cos 7 \theta_{0}-(5 q+m-m q) \cos 5 \theta_{0} \\
& +(7 q+2+3 m-2 m q) \cos 4 \theta_{0}+\left(m^{2}+n q-3 m-6+2 n-6 q\right) \cos 3 \theta_{0} \\
& +\left(2 m q-6 n+2 q+6-3 m^{2}-3 n q-m\right) \cos 2 \theta_{0} \\
& +\left(8 m+3 m^{2}+3 n q-2+6 n-m q\right) \cos \theta_{0}+\left(-6 m-2 n-m^{2}-n q\right), \\
& A_{3}=-q \cos 7 \theta_{0}+(m q+3 q) \cos 6 \theta_{0}+(2 n+m-4 m q) \cos 5 \theta_{0} \\
& +(5 m q-2 q-6 n-2-3 m) \cos 4 \theta_{0}+\left(4 m-m^{2}-n q+3 q+6+4 n\right) \cos 3 \theta_{0} \\
& +\left(2 m^{2}+2 n q-2 m-4-5 m q-q+4 n\right) \cos 2 \theta_{0} \\
& +\left(8 m q-5 m-6+m^{2}+n q\right) \cos \theta_{0}+\left(5 m-m q+2 n+6-2 m^{2}-2 n q\right), \\
& A_{4}=-2 q \cos 10 \theta_{0}+(m q+6 q) \cos 9 \theta_{0}+(2 n-4 q-3 m q) \cos 8 \theta_{0} \\
& +(2 m q-2 q-6 n) \cos 7 \theta_{0}+(2 m+4 n+6 q+2 m q) \cos 6 \theta_{0} \\
& +\left(4 n-3 m q-2 q-m^{2}-n q-6 m\right) \cos 5 \theta_{0}+\left(3 m^{2}+3 n q+m q\right. \\
& +4 m-6 n-2+q) \cos 4 \theta_{0}+\left(5 m-m q-2 m^{2} 2 n-2 n q+6-3 q\right) \cos 3 \theta_{0} \\
& +\left(-9 m-2 m^{2}-2 n q-4+2 m q+m+q\right) \cos 2 \theta_{0} \\
& +\left(3 m^{2}+3 n q-4+m q+q+m+2 q-2\right) \cos \theta_{0} \\
& +\left(6+2 m-2 m q-m^{2}-n q-3 q\right), \\
& A_{5}=(m q+4 q) \cos 8 \theta_{0}+(2 q-2 m q+2 n) \cos 7 \theta_{0}+(-7 q-m q-4 n) \cos 6 \theta_{0} \\
& +(4 m q-2 n+2 m) \cos 5 \theta_{0}-2 q \cos 9 \theta_{0} \\
& +\left(-2 m q+3 q-3 m+8 n-m^{2}-n q\right) \cos 4 \theta_{0} \\
& +\left(-4-4 m+2 q-2 n+2 m^{2}+2 n q\right) \cos 3 \theta_{0} \\
& +\left(8+8 m+m q-q-m^{2}-n q-4 n\right) \cos 2 \theta_{0} \\
& +\left(6 m+4-2 m^{2}-2 n q-2 q+2 n-4 m q\right) \cos \theta_{0} \\
& +\left(m^{2}+n q-8-5 m+m q+q\right) \text {. }
\end{aligned}
$$

From the theory of the direction of Neimark-Sacker bifurcation and the stability of the bifurcating invariant curve (see Sacker [12,13], Wiggins [11] or Kuznetsov [10]), we have the following theorem.

Theorem 4.2 For system (3.1), if $q^{2}+m q-n-1=0$ holds, then $a(0)<0$ (respectively $>0$ ) implies that a unique and stable (respectively unstable) closed invariant curve bifurcates from $X^{*}$, and the Neimark-Sacker bifurcation in $X^{*}$ is supercritical (respectively subcritical). 
Competing interests

The author declares that she has no competing interests.

\section{Acknowledgements}

The author would like to thank the referees and her supervisor, Doctor Reza Memarbashi, for their useful comments.

Received: 24 October 2014 Accepted: 24 July 2015 Published online: 15 August 2015

\section{References}

1. Eckaus, RS: The stability of dynamic models. Rev. Econ. Stat. 39, 172-182 (1957)

2. Hicks, JR: A Contribution to the Theory of the Trade Cycle. Clarendon, Oxford (1965)

3. Dai, B, Zhang, N: Stability and global attractivity for a class of nonlinear delay difference equations. Discrete Dyn. Nat. Soc. 3, 227-234 (2005)

4. Li, S, Zhang, W: Bifurcations in a second-order difference equation from macroeconomics. J. Differ. Equ. Appl. 2, 365-374 (2007)

5. Memarbashi, R, Ghasemabadi, A: Regarding the dynamics of a third order nonlinear difference equation. Adv. Differ. Equ. 2012, 107 (2012)

6. Sedaghat, H: Nonlinear Difference Equations: Theory with Applications to Social Science Models. Kluwer Academic, Dordrecht (2003)

7. Wen, G, Xu, D, Han, X: On creation of Hopf bifurcations in discrete-time nonlinear systems. Chaos 12, $350-355$ (2002)

8. Wen, G, Xu, D: Implicit criteria of eigenvalue assignment and transversality for bifurcation control in four-dimensional maps. Int. J. Bifurc. Chaos 14, 3489-3503 (2004)

9. Stuart, AM, Humphries, AR: Dynamical Systems and Numerical Analysis. Cambridge University Press, Cambridge (1998)

10. Kuznetsov, YA: Elements of Applied Bifurcation Theory. Springer, New York (1998)

11. Wiggins, S: Introduction to Applied Nonlinear Dynamical Systems and Chaos. Springer, New York (1990)

12. Sacker, RJ, Von Bremen, HF: Bifurcation of maps and cycling in genetic systems. Fields Inst. Commun. 42, 305-311 (2004)

13. Sacker, RJ: On Invariant Surfaces and Bifurcation of Periodic Solutions of Ordinary Differential equations. Technical report IMM-NYU 333, New York University (1964)

\section{Submit your manuscript to a SpringerOpen ${ }^{\circ}$ journal and benefit from:}

- Convenient online submission

Rigorous peer review

- Immediate publication on acceptance

- Open access: articles freely available online

- High visibility within the field

- Retaining the copyright to your article 